\title{
Result Analysis-Edge-Preserving Decomposition-based Single Image Haze Removal
}

\author{
Puja Rani Boipai \\ M.Tech Scholar \\ Department of Electronics \& \\ Communication Engineering, \\ IES, Bhopal, India
}

\author{
Sonulal \\ Asst. Prof. \\ Department of Electronics \& \\ Communication Engineering, \\ IES, Bhopal, India
}

\author{
Deepak Mishra \\ Asst. Prof. \\ Department of Electronics \& \\ Communication Engineering, \\ IES, Bhopal, India
}

\begin{abstract}
With the increase in industrial production and human activities, the concentration of atmospheric particulate matter (PM) is substantial increased, leading to fog and haze occurs more frequently. Limited visibility caused by suspended particles in the air, such as fog and haze, is a major problem for many applications of computer vision. The captured scenes by such computer vision systems suffer from poor visibility, low contrast, dimmed brightness, low luminance and distorted color, which makes detection of objects within the scene more difficult. Therefore visibility improvement, contrast and features enhancement of images and videos captured in bad weather, also called as dehazing, and is an inevitable task. Furthermore, estimated actual weather condition is valuable information to invoke corresponding approaches.
\end{abstract}

\section{Keywords}

Single image haze removal, edge-preserving smoothing, weighted guided image filtering, minimal color channel.

\section{INTRODUCTION}

A main objective in dehaze analysis is improvement of visibility and recovery of colours, as if imaging is completed in clear conditions. Then pc vision and human vision will method on such improved pictures for numerous applications, like long vary police work. The requirement for image improvement stems from the actual fact that the atmosphere is rarely freed from particles. Even simply pure air, the visibility has been found to be between $277 \mathrm{~km}$ [1] to $348 \mathrm{~km}$ [2], [3], not considering the curvature of the earth's surface. However, real visual ranges are a lot of not up to this theoretical worth. The international visibility codes for meteorological vary rates visibilities between beneath $50 \mathrm{~m}$ up to over $50 \mathrm{~km}$ for exceptionally clear air. These codes are found to reflect a convenient scale for visual ranges within the daily work of meteorologists. Recently, haze removal through single image attracted a lot of interest and created important progresses because of its broad applications. Several single image haze removal algorithms were planned. Supported an observation that a haze-free image has higher distinction than its haze image, a stimulating single image haze removal algorithmic rule was planned in [5] by increasing the native distinction of the fixed up image.

One of the foremost basic kinds of pollution - haze - is that the major reason behind reduced visibility in several international cities and scenic areas. Haze is caused once daylight encounters small material within the air that reduces the clarity and color of what we tend to see, and significantly throughout wet conditions. Several outside laptop vision applications like video police work, object detection, seeing, tracking; intelligent vehicles and remote sensing systems etc. assume that the input pictures have clear visibility.

Many outdoor computer vision applications like video surveillance, object detection, object recognition, tracking, intelligent vehicles and remote sensing systems etc. assume that the input images have clear visibility. Unfortunately, this is not always true in many situations, in particular, haze and fog weather occurring more and more frequently. Outdoor images or videos are usually degraded by light scattering and absorbing from the aerosols, such as dust, mist, and fumes in the atmosphere, here regarded as haze.

\section{THEORY}

Digital pictures are composed of pixels (short for image elements). every pixel represents the colour (or gray level for black and white photos) at one purpose within the image, thus a pixel is sort of a small dot of a specific color. By measure the colour of a picture at an oversized range of points, we will create a digital approximation of the image from that a copy of the first will be reconstructed. Pixels are a little like grain particles during a standard photographic image, however arranged during a regular pattern of rows and columns and store data somewhat differently. A digital image could be a rectangular array of pixels typically known as a bitmap.

A color image is created from pixels every of that holds 3 numbers similar to the red, green, and blue levels of the image at a specific location. Red, green, and blue (sometimes mentioned as RGB) are the first colours for mixing lightthese alleged additive primary colours are totally different from the subtractive primary colours used for mix paints (cyan, magenta, and yellow). Any color will be created by mix the proper amounts of red, green, and blue lightweight. Assuming 256 levels for every primary, every color pixel will be kept in 3 bytes ( 24 bits) of memory. This corresponds to roughly 16.7 million totally different possible colours. Note that for pictures of a similar size, a black and white version can use 3 times less memory than a color version.

Some color pictures are created using a restricted palette of colours, usually 256 totally different colours. These pictures are observed as indexed color pictures because the info for every pixel consists of a palette index indicating that of the colours within the palette applies thereto pixel. There are many issues with using indexed color to represent photographic pictures. First, if the image contains additional totally different colours than are within the palette, techniques like dithering should be applied to represent the missing colours and this degrades the image. Second, combining 2 indexed color pictures that use totally different palettes or maybe retouching a part of one indexed color image creates issues due to the restricted range of available colours.

Binary pictures use only one bit to represent every pixel. 
Since a little will only exist in 2 states - on or off, each pixel during a binary image should be one among 2 colours, typically black or white. This inability to represent intermediate reminder gray is what limits their usefulness in dealing with photographic pictures.

Most applications in pc vision and computer graphics involve the idea of image filtering to reduce noise and/or extract useful image structures. Easy specific linear translationinvariant (LTI) filters like Gaussian filter, Laplacian filter, and Sobel filter are wide utilized in image blurring/sharpening, edge detection, and have extraction [3]. LTI filtering additionally includes the method of resolution a Poisson Equation, like in high dynamic range (HDR) compression [4], image stitching [5], and image matting [6], wherever the filtering kernel is implicitly outlined by the inverse of a consistent Laplacian matrix. The kernels of LTI filters are spatially invariant and independent of any image content. However in several cases, we tend to might want to include further info from a given guidance image during the filtering method.

\section{METHODOLOGY}

\subsection{Image dehazing method}

\subsubsection{Estimating the Transmission and}

\section{Atmospheric Light}

Haze mainly affects the low-frequency component of haze image. Consequently, haze removal across the whole image is approximated and simplified by removing the haze in the lowfrequency component of the degraded image. The specific steps of our algorithm to obtain the transmission map are as follows: Firstly, apply two-dimensional Haar wavelet transform to extract the low-frequency component $\mathrm{I}_{\mathrm{L}}$ and other high-frequency components of the haze image I.

Then, dark channel prior and haze imaging model are adopted to calculate the transmission map $\mathrm{tL}$ of $\mathrm{I}_{\mathrm{L}}$.

Finally, employ guided filter to refine $t_{L}$ In this step, $I_{L}$ is employed as the guided image, and the transmission map $t_{L}$ is treated as the input image. We could get the final refined transmission map $\mathrm{t}_{\mathrm{L}}$

As discussed in this paper, the atmospheric light can be determined based on the dark channel. The top $0.1 \%$ brightest pixels are picked in the dark channel of $\mathrm{I}_{\mathrm{L}}$, and then among all of these pixels, the pixel with the highest intensity in IL is used as $A_{L}$.

\subsubsection{Recovering the Scene Radiance}

Based on the transmission map $\mathrm{t}_{\mathrm{L}}$, atmospheric light $\mathrm{AL}$ of the haze low-frequency image $I_{L}$ and the haze imaging model, we can recover the scene radiance by taking the steps as follows:

When the transmission $t(x)$ is close to zero, the direct attenuation term $J(x) t(x)$ could be very close to zero. The directly recovered image $\mathrm{J}$ is prone to noise; hence the transmission $\mathrm{t}(\mathrm{x})$ must be restricted to a lower bound t0 to make the haze-free image natural by preserving a small amount of haze in the dense haze areas. In this work, a typical value of $t_{0}$ is 0.1 . Now the haze free low-frequency image could be recovered by

$$
J_{L}(x)=\frac{I_{L}(x)-A_{L}}{\max \left(t_{L}(x), t_{0)}\right.}+A_{L}
$$

Where $\mathrm{J}_{\mathrm{L}}$ denotes the recovered haze-free low-frequency part of the haze image I, like the image within the right facet, then, supported the haze-free low-frequency image JL and different high frequencies obtained by Haar wavelet transform. We will reconstruct the scene radiance $\mathbf{J}$ with inverse Haar wavelet transform, during which the first low-frequency part $\mathrm{I}_{\mathrm{L}}$ is replaced by the haze-free low-frequency part JL.

A novel single image dehazing technique supported dark channel previous is planned. The first dark channel previous based technique employs soft matting algorithmic rule to refine the transmission map that is time-consuming. Recently, guided filter, that is time-saving, has been introduced to perform a similar perform as soft matting. In our work, supported the observation that haze mainly has an impact on the low-frequency part of a picture, by using guided filter rather than soft matting algorithmic rule to refine the transmission map of the low-frequency part of haze image extracted by Haar wavelet transform, the runtime price is dramatically decreased. Experimental results show that the planned algorithmic rule restores the image faster whereas maintaining relatively excellent performance compared with the Dark Channel previous with guided Filter based mostly technique that permits this algorithmic rule to be applied to the time period applications like obstacle detection and surveillance.

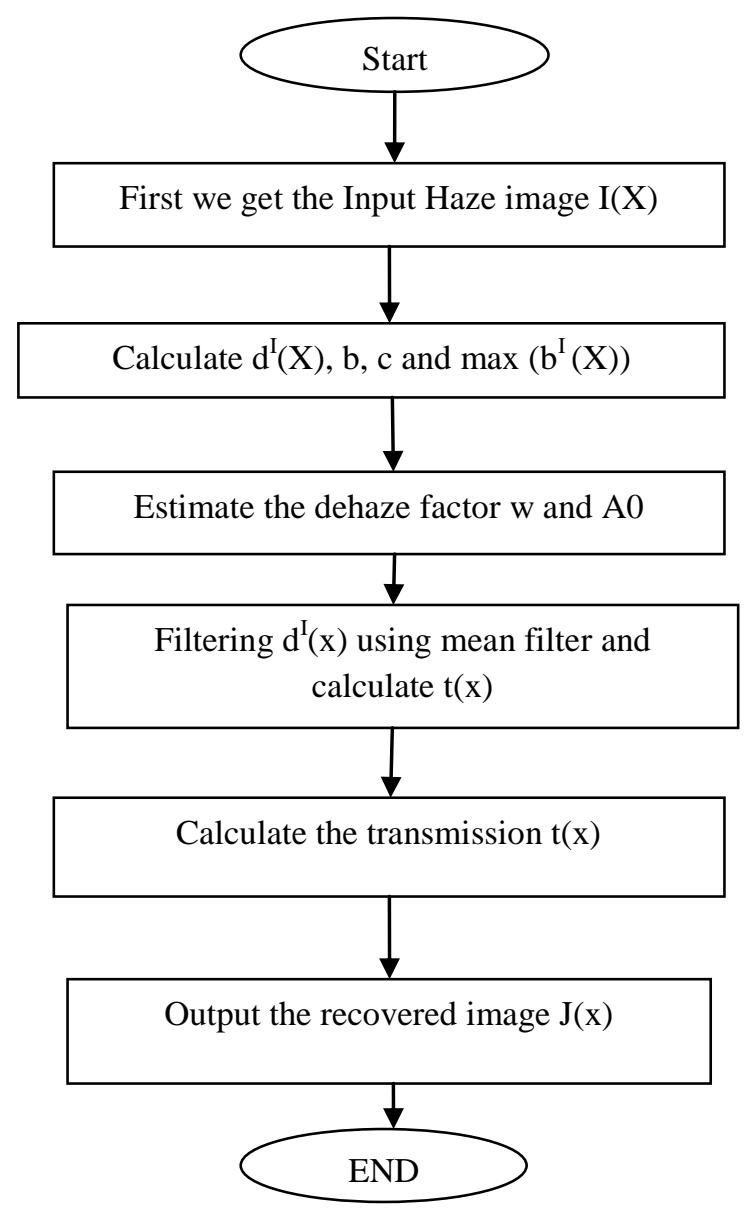

Fig.1: Algorithm to Find the Haze Free Image

\section{RESULT}

In the analysis of edge preserving of image we can see that images with the haze are analyzed and gives the following results. 


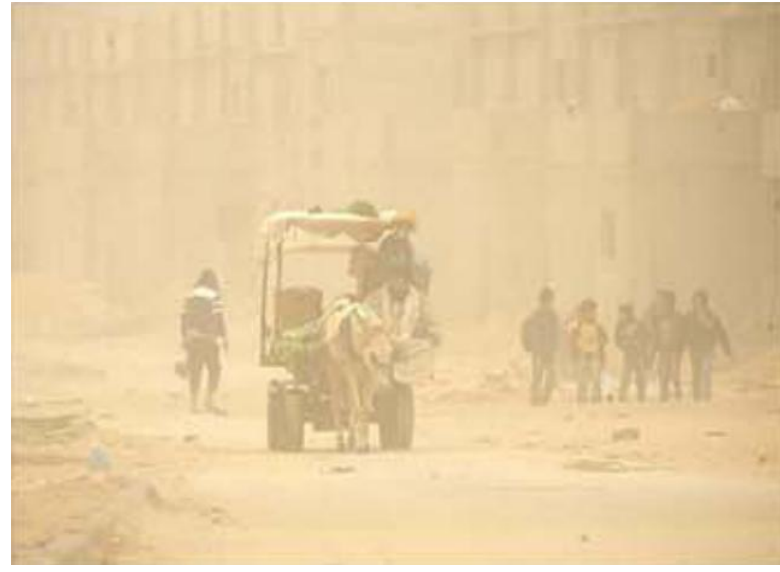

Fig.2 Input Image

As show in above Figure 2 is input image. In the above figure we can see the hazy image after taking input haze image we proposed the haze removal. In above input object the dark channel estimation algorithm is applied and the dark region and light intensity of object is found. After the collecting dark information in object we mark it as show in figure 3 .

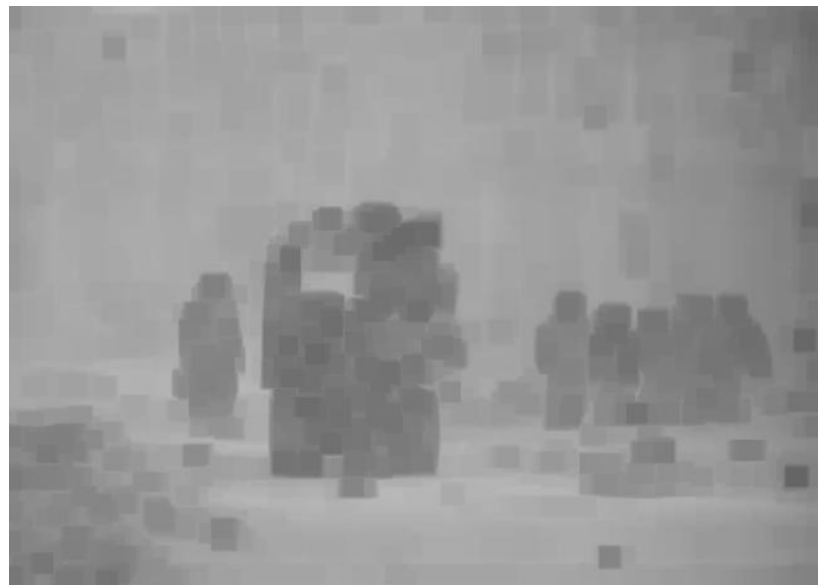

Fig 3: Result image in dark form after haze removal

The fig. 3 shows the first output is a dark form of image after haze removal. After dark channel estimation then the atmospheric estimation and transient estimation algorithms are applied to calculate variations as shown in figure 3 .

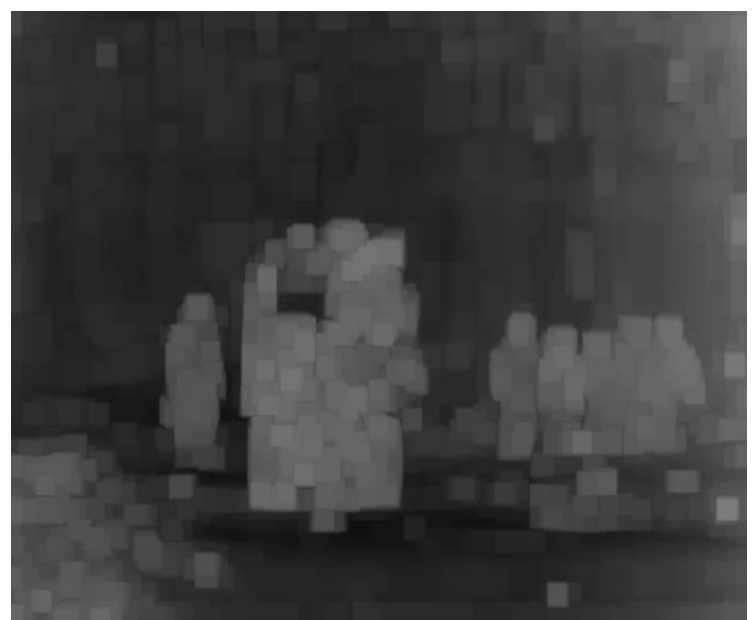

Fig 4: Result image in transient form after haze removal
The estimate of pixel value in dark region and the atmospheric variation estimate is the estimation of transmission obtained as the transient image figure. 4 .

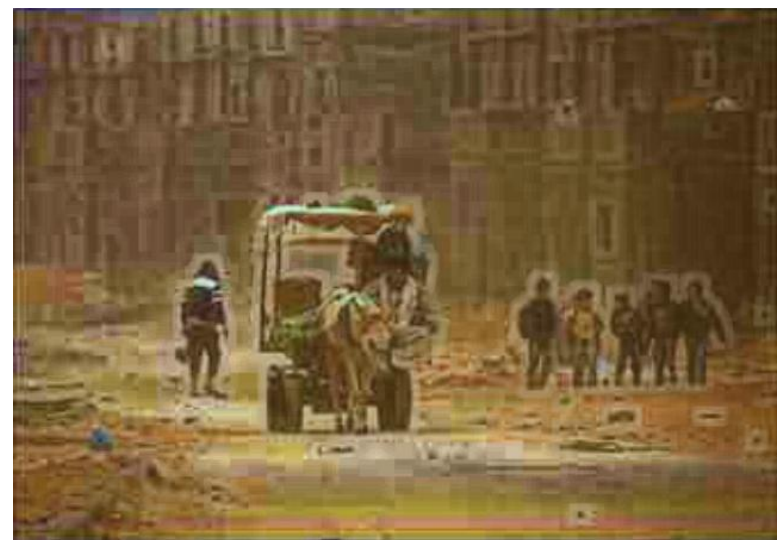

Fig 5: Result in Radiant image after haze removal

Fig.5 is the radiant or dehazed image of the object obtained after further optimization by smoothening the transient image.

Table .1 Result Analysis

\begin{tabular}{|c|c|c|}
\hline Size & Running Time & $\begin{array}{l}\text { Quality } \\
\text { (PSNR) }\end{array}$ \\
\hline $500 \times 500$ & 2.3 second & $38 \mathrm{~dB}$ \\
\hline $800 \times 1000$ & 4.5 second & $36 \mathrm{~dB}$ \\
\hline $1680 \times 1680$ & 12 second & $27 \mathrm{~dB}$ \\
\hline
\end{tabular}

\section{CONCLUSION}

In this paper result analysis of single image haze removal techniques. Haze removal is planned by means that of the dark channel previous technique and estimation of the atmospherical lightweight technique. Dark channel region is calculated by using dark channel algorithmic rule then the atmospherical variation is calculated thereon dark channel region. The planned algorithmic rule may be a new framework for single image haze removal that is from the Koschmiedars law without using any previous.

\section{REFERENCES}

[1] Zhengguo Li, and Jinghong Zheng, "Edge-Preserving Decomposition-Based Single Image Haze Removal", Ieee Transactions On Image Processing, Vol. 24, No. 12, December 2015

[2] Yuan-Kai Wang and Ching-Tang Fan "Single Image Defogging by Multiscale Depth Fusion", IEEE Transactions On Image Processing, Vol. X, No. X, Month 2014.

[3] Faming Fang, Fang Li, and Tieyong Zeng "Single Image Dehazing and Denoising: A Fast Variational Approach", 2014 Society for Industrial and Applied Mathematics.

[4] Codruta Orniana Ancuti and Cosmin Ancuti "Single Image Dehazing by Multi-Scale Fusion". IEEE Transactions On Image Processing, Vol. 22, No. 8, August 2013.

[5] Jing Yu, Qingmin Liao "Fast Single Image Fog Removal 
Using Edge-Preserving Smoothing", 978-1-4577-05397/11/\$26.00 @2011 IEEE

[6] Raanan Fatta "Single Image Dehazing", Hebrew University of Jerusalem, Israel.

[7] S. G. Narasimhan and S. K. Nayar, "Chromatic framework for vision in bad weather," in Proc. IEEE Conf. Comput. Vis. Pattern Recognit. (CVPR), Hilton Head Island, SC, USA, Jun. 2000, pp. 598-605.

[8] S. G. Narasimhan and S. K. Nayar, "Contrast restoration of weather degraded images," IEEE Trans. Pattern Anal. Mach. Learn., vol. 25, no. 6, pp. 713-724, Jun. 2003.

[9] J. Kopf et al., "Deep photo: Model-based photograph enhancement and viewing," ACM Trans. Graph., vol. 27, no. 5, pp. 1-10, May 2008.
[10] K. He, J. Sun, and X. Tang, "Single image haze removal using dark channel prior," IEEE Trans. Pattern Anal. Mach. Intell., vol. 33, no. 12, pp. 2341-2353, Dec. 2011.

[11] X. Y. Lv, W. Chen, and I. Shen, "Real-time dehazing for image and video," in Proc. 18th Pacific Conf. Comput. Graph. Appl., Hangzhou, China, Sep. 2010, pp. 62-69.

[12] J. Pang, O. C. Au, and Z. Guo, "Improved single image dehazing using guided filter," in Proc. APSIPA ASC, Xi'an, China, 2011, pp. 1-4.

[13] K. He, J. Sun, and X. Tang, "Guided image filtering," IEEE Trans. Pattern Anal. Mach. Intell., vol. 35, no. 6, pp. 1397-1409, Jun. 2013.

[14] Z. Li, J. Zheng, Z. Zhu, W. Yao, and S. Wu, "Weighted guided image filtering," IEEE Trans. Image Process., vol. 24, no. 1, pp. 120-129, Jan. 2015. 